\title{
Editorial
}

\section{Left-right asymmetry in plants and animals: a gold mine for research}

\author{
Menno Schilthuizen ${ }^{1,2,4}$, Barbara Gravendee ${ }^{1,2,3}$ \\ ${ }^{1}$ Netherlands Centre for Biodiversity Naturalis, Darwinweg 2, 2333 CR Leiden, The Netherlands \\ ${ }^{2}$ Institute Biology Leiden, Leiden University, P.O. Box 9516, 2300 RA Leiden, The Netherlands \\ ${ }^{3}$ University of Applied Sciences Leiden, P.O. Box 382, 2300 AJ Leiden, The Netherlands \\ ${ }^{4}$ E-mail: menno.schilthuizen@ncbnaturalis.nl
}

Key words: chirality, development, evolution, evo-devo

\begin{abstract}
Left-right asymmetry patterns in the body shapes of animals and plants have been a continuous source of interest among biologists. Recently, inroads have been made to developing a coherent research programme that makes use of the unique fact that chiral patterns may be studied (and generalities deduced) by comparisons across many unrelated groups, even across Kingdoms. The papers delivered at the symposium 'Evolution of Chirality' during the 2011 European congress of evolutionary biology (ESEB) provide examples of the various research programs that are currently developing within this field. The present paper provides a summary of the symposium, an introduction to this Special Issue of Contributions to Zoology, as well as suggestions for further collaboration among left-right asymmetry researchers.
\end{abstract}

\section{Introduction}

Throughout evolution, developmental systems for whole and partial body forms in animals and plants have frequently converged to bilateral symmetry, due to the requirements to balance forces on bodies or body parts exposed to gravity or to the stresses of a moving medium (Ludwig, 1932; McManus, 2002). This may be due either to their living in a moving medium - air or water - themselves, or because they are mobile organisms living in a static medium. In fact, organisms that are too small or live in an environment that is too protected to be affected by such stresses, and, hence, develop in an amorphic manner, are very few. Consequently, we tend to consider bilateral symmetry as the default developmental mode for most multicellular animals and even for the gross structure of plants and their parts (e.g., flowers, leaves). Yet, secondary asymmetry among organisms that normally develop symmetrically is very common
(Palmer, 2004). It frequently affects internal parts (placed in a protected internal 'environment', these are less forced into symmetry by the need to balance forces), but also external parts, and sometimes even the entire body. In animals, oft-cited examples are snails with their obvious whole-body asymmetry, fiddler crabs with one claw much larger than the other, and the arrangement of humans' internal organs. In plants, individual flowers can be asymmetrical by skewed positions of the female and/or male reproductive organs or twisting of the sepals and/or petals or fruits into a certain direction only (see Fig. 1 for some examples). Whole inflorescences, stems, leaves or modified leaf tips such as tendrils can also be twisted to either the left or the right (Kihara, 1972). It was recently discovered that some of these adaptations promote outcrossing (Jesson and Barrett, 2002; Iwata et al., 2012).

As has been emphasised by Palmer (1996, 2004), unlike most morphological traits, symmetry and asymmetry in body shape can be studied in a meaningful way across many unrelated taxa. Its binary nature allows the trait to be scored simply and unambiguously in wide ranges of taxa, which makes it possible to draw general conclusions on the evolution of a key developmental trait; something that is normally almost impossible in evo-devo studies.

Over the past few years, the Netherlands Centre for Biodiversity Naturalis (NCB Naturalis) has been capitalising on the joint morphological knowledge held by its taxonomists and contained in its taxonomic collections by initiating a research program on left-right asymmetry. One activity has been the organisation of a symposium on the Evolution of Chirality at the 13th Congress of the European Society for Evolutionary Biology (ESEB) in Tübingen, Germany. We are very 

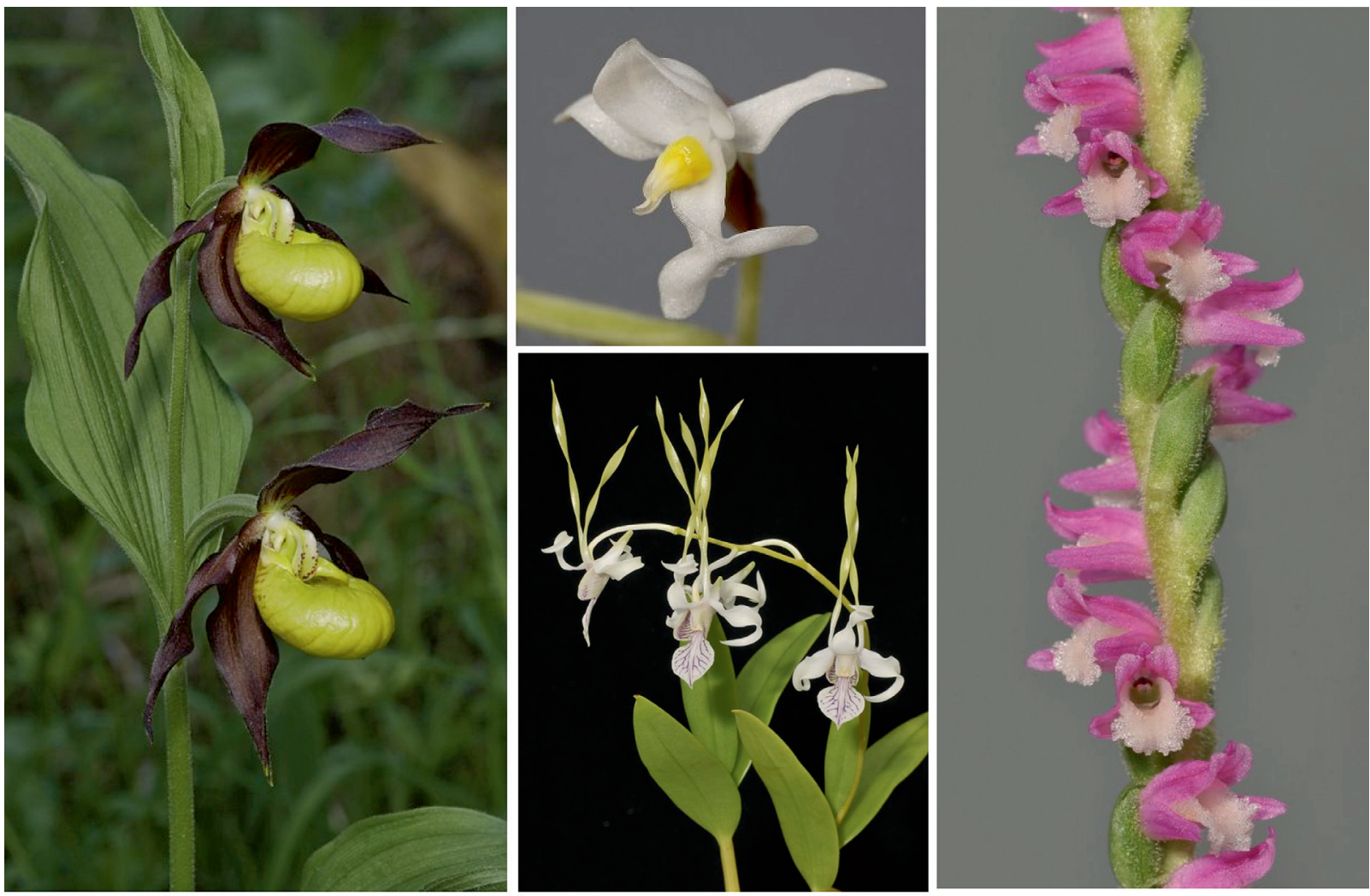

Fig. 1. Examples of left-right asymmetry in orchids. From left to right: Cypripedium calceolus with twisted tepals, Ludisia discolor with twisted anther, Dendrobium antennatum with twisted petals and Spiranthes sinensis with twisted inflorescence axis.

happy that Contributions to Zoology has been keen to publish the proceedings of this symposium, which we hope will further consolidate the position of asymmetry as a unifying subject across disciplines, and will serve to generate novel research avenues for the future.

\section{The symposium papers}

Three of the papers in the Special Issue approach left-right asymmetry in a bottom-up fashion, examining patterns within individual species. The paper by Przybyłowicz et al. (2012) on chemical mimicry of the European lady's slipper orchid Cypripedium calceolus, is a novel attempt to place flower chirality in the context of pollination (see Jesson and Barrett (2003) for other ways in which floral chirality interacts with pollination). In some (but not all) Cypripedium species (among which $C$. calceolus) the tepals all coil in the same direction, regardless of whether the tepal is on the right or on the left of the flower's midline (Welch, 1998). The resultant asymmetry might play a role in insect deception by distorting floral symmetry, as bees have more difficulty memorizing asymmetrical flowers than symmetrical ones (Rodríguez et al., 2004), and this may result in longer-lasting deception. The paper by Przybyłowicz et al. (2012) works towards investigating this hypothesis by first assessing the chemical cues used by flower visitors.

Lang and Orgogozo's (2012) work on the mating behaviour in a Drosophila species with asymmetric genitalia similarly approaches chirality from a sexual viewpoint. Genital asymmetry is exceedingly common among insects (Schilthuizen, 2007), with recurrent phylogenetic patterns in different taxa. Huber et al. (2007) suggested that it may be driven by the evolution of male-dominant mating positions, but few attempts have been made to test this hypothesis experimentally. Lang and Orgogozo (2012) were fortunate in discovering a symmetric mutant in a lab stock of D. pachea, which allowed them to perform ground- 
breaking experiments on the effect of genital (a)symmetry on mating behaviour. They found that the symmetric males were less able to couple properly with females. Also, the mating position (slightly slanted in the wildtype) was less stable.

The paper by Schilthuizen et al. (2012) also investigates a single species, the chirally dimorphic tropical tree snail Amphidromus inversus. Earlier investigations (Schilthuizen et al., 2007) had shown that dextral and sinistral enantiomorphs differ in mating behaviour (they prefer to mate with individuals of opposite coiling), and in shell morphology (subtle differences in the shell proportions; Schilthuizen and Haase, 2010). However, whether differences in dispersal or resting sites exist between the two coiling morphs remained to be investigated. In their paper, Schilthuizen et al. (2012) show that this does not seem to be the case, an indication that the disassortative mate choice may indeed be the main factor maintaining this dimorphism.

The last two papers take a top-down approach to asymmetry, looking for global patterns. Hoso (2012) does so by investigating the island biogeography of sinistral coiling in snails. Theory predicts that speciation driven by coil reversal (Gittenberger, 1988), though unlikely in itself, may be aided by small population size (van Batenburg and Gittenberger, 1996). This led Hoso (2012) to hypothesise that higher proportions of coil-reversed species should be found on oceanic islands (which have smaller population sizes and more frequent population bottlenecks) compared with the mainland. Based on an analysis of chirality and biogeography patterns in almost a thousand snail genera, Hoso (2012) concludes that this hypothesis is indeed supported.

Palmer (2012), finally, has graced this Special Issue with an important review paper on the developmental mechanisms responsible for populations with both left- and right-directed asymmetries. Two patterns exist in nature, and a different developmental threshold model appears to apply to each. First, populations in which, besides symmetric individuals, conspicuous (dextral or sinistral) asymmetries exist, may do so due to a threshold system with lateral inhibition of one side, as soon as the other side has differentiated. Second, symmetric populations with relatively rare asymmetric individuals are more likely to be caused by an ontogenetic model without such lateral inhibition. The models are discussed in the light of the recently-reported case of leg asymmetry in the dance fly, Empis jaschhoforum (Daugeron et al., 2011).

\section{Outlook}

Though modest in size, we hope this collection of papers will emphasise the benefits of tying together different asymmetry research strands. After all, if there is one developmental trait that is eminently suited to perform a unifying role in evo-devo research, it is chirality. As pointed out in Palmer's (2012) paper, coherent studies of morphological asymmetries have already yielded valuable insights into how development evolves: Are genes leaders or followers in evolution? Does behaviour play a role in the generation of novel phenotypes? Do homologous traits in different taxa have homologous development? Do developmental pathways become more robust - less vulnerable to disruption - over evolutionary time? We could, however, envisage additional ways in which left-right asymmetry research may bring progress in our understanding of evolution.

For example, as pointed out in Lang and Orgogozo's (2012) paper, genital asymmetry is a very widespread phenomenon in insects, but also in most other animal taxa with internal fertilization (Schilthuizen, in prep.). This phenomenon is interesting for several reasons. First, it throws a new light on the assumption that symmetry is a preferred sexual signal (van Dongen, 2006). Second, it highlights the reproductive system as unique among animal organ systems in that it can apparently alternate between strictly symmetric and strongly asymmetric development. Understanding how this is effected could be of relevance for diagnosing and treating congenital symmetry defects in humans.

Exciting advances in genetic and experimental research on left-right asymmetry of plants have been made over the past 40 years. We now know for instance that the twisted growth of corkscrew hazelnut and asymmetry of flowers of Heteranthera seem controlled by a single gene (Smith and Mehlenbacher, 1996; Jesson and Barrett, 2002). In the next decade, we will undoubtedly discover the identity of these genes using Next Generation sequencing. This will shed further light on the genetic basis of evolutionary and economically important (a)symmetric plant traits and their adaptive values.

\section{Acknowledgements}

We thank all the authors of papers in the Special Issue as well as the speakers at the ESEB symposium. Moreover, we thank Nico Michiels and his team at ESEB for allowing us to organise the symposium and for being so accommodating. Generous 
financial support for keynote speakers Richard A. Palmer and Spencer Barrett was provided by the SWO granting scheme of NCB Naturalis. Rogier R. van Vugt (Hortus Botanicus of Leiden University) is thanked for providing the illustrating photographs.

\section{References}

Batenburg FHD van, Gittenberger E. 1996. Ease of fixation of a change in coiling: computer experiments on chirality in snails. Heredity 76: 278-286.

Daugeron C, Plant A, Winkler I, Stark A, Baylac M. 2010. Extreme male leg polymorphic asymmetry in a new Empididine dance fly (Diptera: Empidididae). Biology Letters 7: 1114.

Dongen S van. 2006. Fluctuating asymmetry and developmental instability in evolutionary biology: past, present and future. Journal of Evolutionary Biology 19: 1727-1743.

Gittenberger E. 1988. Sympatric speciation in snails - A largely neglected model. Evolution 42:826-828.

Hoso M. 2012. Non-adaptive speciation of snails by left-right reversal is facilitated on oceanic islands. Contributions to Zoology 81: 79-85.

Huber BA, Sinclair BJ, Schmitt M. 2007. The evolution of asymmetric genitalia in spiders and insects. Biological Reviews 82: 647-698.

Iwata T, Nagasaki O, Ishii HS, Ushimaru A. 2012. New Phytologist 193: 196-203.

Jesson LK, Barrett SCH. 2002. The genetics of mirror-image flowers. Proceedings of the Royal Society of London B series 269: 1835-1839.

Jesson LK, Barrett SCH. 2003. The comparative biology of mirror-image flowers. International Journal of Plant Science 164: S237-S249.

Kihara H. 1972. Right- and Left-handedness in Plants. A Review. Seiken Zihô 23: 1-37.

Lang M, Orgogozo V. 2012. Distinct copulation positions in Drosophila pachea males with symmetric or asymmetric external genitalia. Contributions to Zoology 81: 87-94.
Ludwig W. 1932. Das Rechts-Links-Problem im Tierreich und beim Menschen. Berlin: Springer.

McManus C. 2002. Right Hand, Left Hand; The Origins of Asymmetries in Brains, Bodies, Atoms, and Cultures. Cambridge: Harvard University Press.

Palmer AR. 1996. From symmetry to asymmetry: phylogenetic patterns of asymmetry variation in animals and their evolutionary significance. Proceedings of the National Academy of Sciences USA 93: 14279-14286.

Palmer AR. 2004. Symmetry breaking and the evolution of development. Science 306: 828-833.

Palmer AR. 2012. Developmental origins of normal and anomalous random right-left asymmetry: lateral inhibition versus developmental error in a threshold trait. Contributions to Zoology 81: 111-124.

Przybyłowicz T, Roessingh P, Groot AT, Biesmeijer JC, Oostermeijer JGB, Chittka L, Gravendeel B. 2012. Possible chemical mimicry of the European lady's slipper orchid (Cypripedium calceolus). Contributions to Zoology 81: 103-110.

Rodríguez I, Gumbert A, Hempel de Ibarra N, Kunze J, Giurfa M. 2004. Symmetry is in the eye of the 'beeholder': innate preference for bilateral symmetry in flower-naïve bumblebees. Naturwissenschaften 91: 374-377.

Schilthuizen M. 2007. The evolution of chirally dimorphic insect genitalia. Tijdschrift voor Entomologie 150: 347-354.

Schilthuizen M, Craze PG, Cabanban AS, Davison A, Gittenberger E, Stone J, Scott BJ. 2007. Sexual selection maintains whole-body chiral dimorphism. Journal of Evolutionary Biology 20: 1941-1949.

Schilthuizen M, Haase M. 2010. Disentangling true shape differences and experimenter bias: are dextral and sinistral snail shells exact mirror images? Journal of Zoology 282: 191-200.

Schilthuizen M, Haase M, Koops K, Looijestijn SM, Hendrikse S. 2012. The ecology of shell shape difference in chirally dimorphic snails. Contributions to Zoology 81: 95-101.

Smith DC, Mehlenbacher SA. 1996. Inheritance of contorted growth in hazelnut. Euphytica 89: 211-213.

Welch CJ. 1998. Some observations concerning the asymmetry of orchid flowers. Malayan Orchid Review 32: 86-92. 